\title{
Primer registro de Rara (Phytotoma rara Molina, 1782) en Isla Riesco, Magallanes
}

\author{
First record of Rufous-tailed plantcutter \\ (Phytotoma rara Molina, 1782) in Riesco Island, Magallanes
}

Javier A. Simonetti ${ }^{1,2}$ \& Gregor J. Stipicic ${ }^{2,3}$

La rara (Phytotoma rara Molina, 1782) es un ave herbivora pequeña, consume preferentemente hojas y frutos, habitando matorrales y bosques además de jardines y huertos (Johnson \& Goodall, 1967; López-Calleja \& Bozinovic, 1999). Considerada poco común en Chile, el límite septentrional de P. rara es Vallenar, alcanzando por el sur hasta el norte de Magallanes, donde sería un visitante estival. Si bien su límite meridional sería Puerto Natales (514' S), existen registros hasta Tierra del Fuego, Ushuahia e islas Malvinas. Sin embargo, su presencia, tanto en Malvinas como Tierra del Fuego, es considerada como accidental en tanto sería un visitante raro y de estado indefinido en la Región de Magallanes (Johnson \& Goodall, 1967; Venegas, 1977; Venegas \& Sieldfeld, 1998; Couve \& Vidal, 2003; Woods \& Woods, 2006).

No obstante, un número creciente de registros al sur de Puerto Natales sugiere que $P$. rara sería un visitante recurrente de la región. De hecho, a lo menos 14 registros al sur de Puerto Natales se han registrado desde 2010 (GBIF, 2016). En este contexto, a mediados de octubre 2013 observamos brevemente a un macho adulto en el arbolado del casco de la Estancia

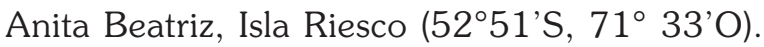
El ejemplar se posó en álamos (Populus alba L.) y lengas (Nothofagus pumilio (Poepp. \& Endl.) Krasser). Posteriormente, el 7 de noviembre de 2015 avistamos durante 10 minutos un macho y una hembra adulta en el mismo sector. Al igual que el ejemplar anterior, la pareja se posó en $P$. alba, N. pumilio y también sobre calafates (Berberis microphylla G. Forst.), arbusto del cual consumían sus flores (Fig. 1). Los días 8 y 9 de noviembre también registramos su presencia, detectadas solamente por su canto.

Los ejemplares observados en la Estancia

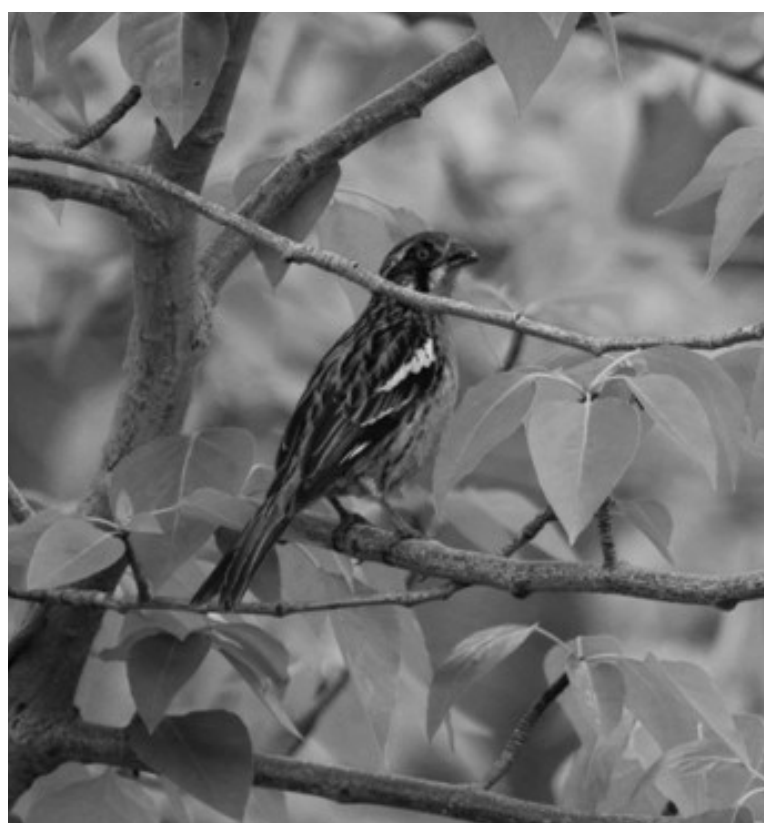

Fig. 1. Macho de Phytotoma rara posado sobre álamo en Estancia Beatriz, Isla Riesco (noviembre 2015).

Departamento de Ciencias Ecológicas, Facultad de Ciencias, Universidad de Chile. $\bowtie$ jsimonet@uchile.cl

2 Asociación Kauyeken, Santiago \& Isla Riesco, Magallanes, Chile.

3 Estancia Anita Beatriz, Isla Riesco, Magallanes, Chile 
Anita Beatriz representan los primeros registros en Isla Riesco. Muestreos previos no le habían detectado (e.g., Markham, 1970; Imberti, 2005; véase además Venegas, 1994). Estos registros se suman a un número creciente de observaciones más meridionales, como se desprende de los registros del Global Biodiversity Information Facility (GBIF, 2016). Registros desde 1980 hasta la actualidad, abarcando desde los $50^{\circ} \mathrm{S}$ al sur, que comprenden numerosos sectores del Parque Nacional Torres del Paine, como el Lago Pehoé, Laguna Grey, Laguna Amarga, Sierra Baguales, así como en Cueva del Milodón, Cerro Guido, Cerro Benítez, la Península Antonio Varas, la Bahía San Juan y registros en Tierra del Fuego. En Argentina existen registros en El Calafate, Parque Nacional Los Glaciares y Ushuahia.

Un $43 \%$ de los registros (sobre un total de 124 observaciones desde los $50^{\circ} \mathrm{S}$ al sur) corresponden a meses de verano (diciembre a febrero), mientras un 56\% ocurre en meses de primavera (septiembre a noviembre), con un solo registro en otoño (mayo). Por la época en que $P$. rara ha sido mayormente registrada, debiera tratarse de un visitante estival que nidifica, ya que ovipone de octubre hasta diciembre (Johnson \& Goodall, 1967).

Considerando el año promedio de los primeros registros en diferentes rangos latitudinales (usando tramos de $0,5^{\circ} \mathrm{S}$ ), los registros al sur de los $50^{\circ} \mathrm{S}$ son progresivamente más modernos (Fig. 2). Esto sugeriría un progresivo avance hacia el sur.

Es poco probable que $P$. rara haya pasado desapercibida con anterioridad. Si bien no es una especie abundante, es particularmente conspicua tanto por su colorido como por su canto. Los ojos rojos brillantes, la frente y cabeza castaña de los machos por ejemplo, sumado a su trino nasal y áspero, precedido de notas tartamudas también son inconfundibles entre las aves chilenas (Jaramillo, 2005). Además, en ocasiones suele ser considerada un ave perjudicial para los cultivos y jardines, por lo que también pudo haber sido detectada antes por estos motivos (e.g., Gigoux, 1940; Silva-Rodríguez

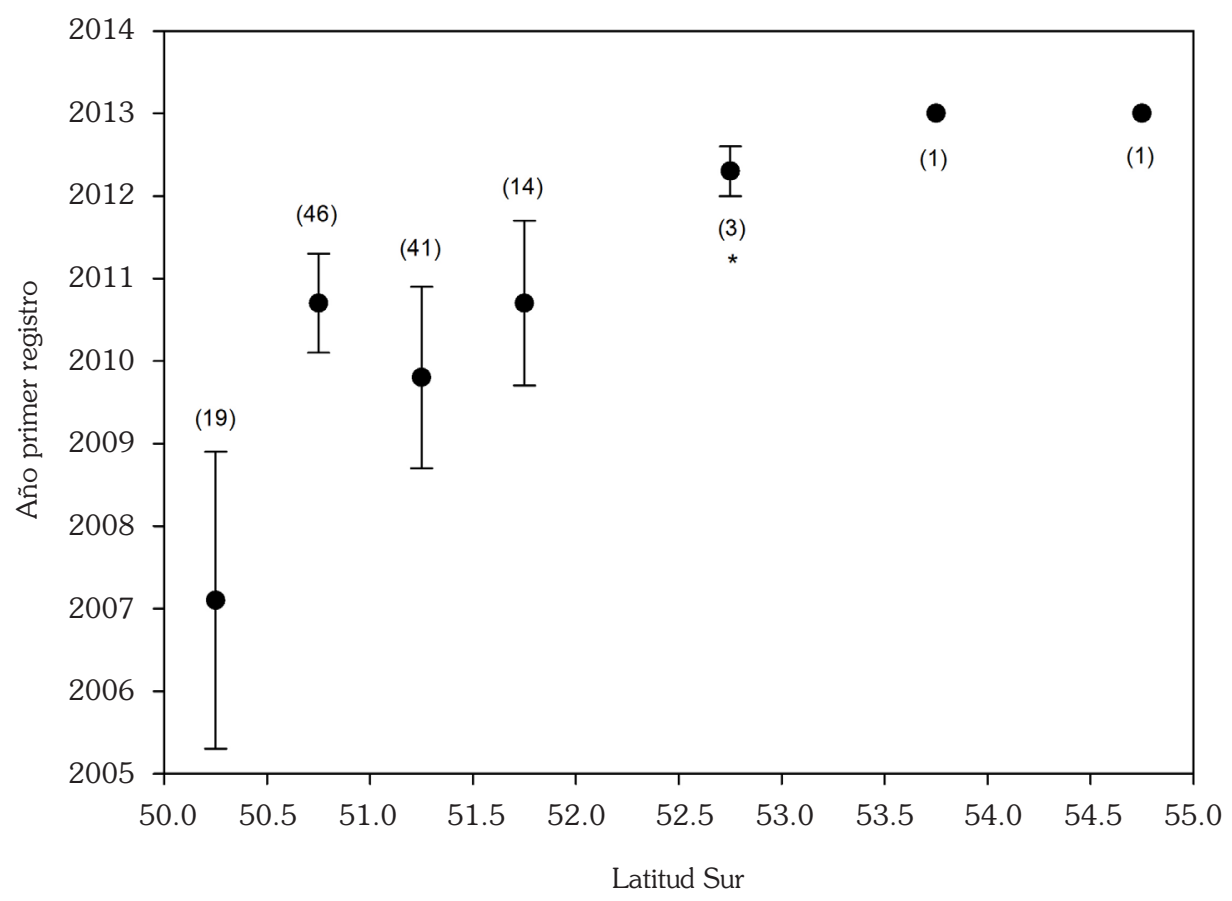

Fig. 2. Tendencia en registros de Phytotoma rara en el sur de Sudamérica. Valores son el año (promedio \pm error estándar) en que ocurre el primer registro en localidades ubicadas en diferentes bandas latitudinales, agrupadas en fajas de $0,5^{\circ}$ de latitud. Como valor de referencia para graficar se presenta el valor de la mediana de cada banda. Los números entre paréntesis son el número de registros. El asterisco indica nuestros la ubicación de nuestros registros. 
et al. 2006).

Los registros repetidos en Isla Riesco así como en otras localidades de Magallanes sugieren que su presencia no sería meramente accidental y que estaría avanzando hacia el sur. Al igual como ocurre con el avance hacia el sur del pato rinconero (Heteronetta atricapilla (Merren, 1841)) y un incremento en la frecuencia de observaciones de la viudita (Colorhamphus parvirostris (Darwin, 1839)) (McGehee et al. 2004, Matus \& Jaramillo, 2008), la presencia y abundancia de P. rara en Isla Riesco así como en otros sectores al sur de Puerto Natales podrían resultar como una respuesta a cambios climáticos. Por ejemplo, el aumento en la temperatura media de las máximas en los últimos 120 años y el incremento en las temperatura media de las mínimas en tiempos recientes (Santana et al. 2009) podrían hacer térmicamente más tolerables las primaveras para $P$. rara y con ello generar los cambios en la distribución y abundancia observada en esta $y$ otras aves y esperada para otros organismos como reptiles (Bonino et al. 2015), por lo que a futuro podrían esperarse cambios en la composición de la fauna magallánica.

\section{LITERATURA CITADA}

Bonino, M. F., Moreno, D. L., Schulte, J. A., \& Cruz. F. B. (2015). Climate change and lizards: changing species' geographic ranges in Patagonia. Regional Environmental Change, 15, 1121-1132.

Couve, E., \& Vidal, C. (2003). Aves de Patagonia, Tierra del Fuego y Península Antártica (1 ${ }^{\mathrm{a}}$ edn.). Punta Arenas, Chile: Fantástico Sur Birding.

Gigoux, E. E. (1940). La rara (Phytotoma rara). Revista Chilena de Historia Natural, 44, 4-7.

GBIF (Global Biodiversity Information Facility) (2016) GBIF Occurrence Download http:// doi.org/10.15468/dl.tsxkaa.

Imberti, S. (2005). Distribución otoñal de aves marinas y terrestres en los canales chilenos. Anales del Instituto de la Patagonia, 33, 21-30.

Jaramillo, A. (2005). Aves de Chile (1 ${ }^{\text {a }}$ edn.). Barcelona, España: Lynx Ediciones.
Johnson, A. W., \& Goodall, J. D. (1967). The birds of Chile and adjacent regions of Argentina, Bolivia and Perú ( $1^{\text {a }}$ edn.). Buenos Aires, Argentina: Platt Establecimientos Gráficos, Vol. 2.

López-Calleja, M. V., \& Bozinovic, F. (1999). Feeding behavior and assimilation efficiency of the Rufous-tailed plantcutter: a small herbivore. Condor, 101, 705-710.

Matus, R., \& Jaramillo, A. (2008). Range extensions and vagrant bird species in the XII Region of Magallanes, Chile. Cotinga, 30, 34-40.

Markham, B. J. (1970). Reconocimiento faunístico del área de los fiordos Toro y Cóndor, isla Riesco, Magallanes. Anales del Instituto de la Patagonia, 1, 41-59.

McGehee, S. M., Rozzi, R., Anderson, C. B., Ippi, S., Vásquez, R. A., \& Woodland, S. (2004). Late-summer presence of the Patagonian Tyrant, Colorhamphus parvirostris (Darwin) on Navarino Island, Cape Horn County, Chile. Anales Instituto Patagonia, 32, 25-33.

Santana, A., Butorovic, N., \& Olave, C. (2009). Variación de la temperatura en Punta Arenas (Chile) en los últimos 120 años. Anales del Instituto de la Patagonia, 37, 85-96.

Silva-Rodríguez, E. A., Ortega-Solís, G. R., \& Jiménez, J. E. (2006). Aves silvestres: actitudes, prácticas y mitos en una localidad rural del sur de Chile. Boletín Chileno de Ornitología, 12, 2-14.

Venegas, C. (1977). Extensión del rango meridional de la rara (Phytotoma rara Mol) hasta Magallanes (Aves: Phytotomidae). Anales del Instituto de la Patagonia, 8, 317-318.

Venegas, C. (1994). Aves de Magallanes (1ª edn.). Punta Arenas, Chile: Ediciones Universidad de Magallanes.

Venegas, C., \& Sieldfeld, W. (1998) Catalogo de los vertebrados de la región de Magallanes y Antártica Chilena ( $1^{\text {a }}$ edn.). Punta Arenas, Chile: Ediciones Universidad de Magallanes.

Woods, R. W. \& Woods, A. (2006). Birds and mammals of the Falkland Islands ( $1^{\text {a }}$ edn.). Hampshire, United Kingdom: WildGuides. 
